\title{
Circuit theory of crossed Andreev reflection
}

\author{
Jan Petter Morten, ${ }^{1, *}$ Arne Brataas, ${ }^{1,2}$ and Wolfgang Belzig ${ }^{3}$ \\ ${ }^{1}$ Department of Physics, Norwegian University of Science and Technology, N-7491 Trondheim, Norway \\ ${ }^{2}$ Centre for Advanced Study, Drammensveien 78, Oslo, N-0271 Norway \\ ${ }^{3}$ Department of Physics, University of Konstanz, D-78457 Konstanz, Germany \\ (Received 21 June 2006; revised manuscript received 11 September 2006; published 18 December 2006)
}

\begin{abstract}
We consider transport in a three-terminal device attached to one superconducting and two normal-metal terminals, using the circuit theory of mesoscopic superconductivity. We compute the nonlocal conductance of the current out of the first normal-metal terminal in response to a bias voltage between the second normal-metal terminal and the superconducting terminal. The nonlocal conductance is given by competing contributions from crossed Andreev reflection and electron cotunneling, and we determine the contribution from each process. The nonlocal conductance vanishes when there is no resistance between the superconducting terminal and the device, in agreement with previous theoretical work. Electron cotunneling dominates when there is a finite resistance between the device and the superconducting reservoir. Dephasing is taken into account, and the characteristic time scale is the particle dwell time. This gives rise to an effective Thouless energy. Both the conductance due to crossed Andreev reflection and electron cotunneling depend strongly on the Thouless energy. We suggest experimental determination of the conductance due to crossed Andreev reflection and electron cotunneling in measurement of both energy and charge flow into one normal-metal terminal in response to a bias voltage between the other normal-metal terminal and the superconductor.
\end{abstract}

DOI: 10.1103/PhysRevB.74.214510

PACS number(s): 74.45.+c, 74.25.Fy, 73.23.-b

\section{INTRODUCTION}

Crossed Andreev reflection ${ }^{1,2}$ transforms an incident electron from one conductor, attached to a superconductor, into a hole in a geometrically separated second attached conductor. In an alternative, equivalent picture, two quasiparticles from two separate conductors are transferred into a superconductor as a Cooper pair. Electrons can also be transferred between the conductors by electron cotunneling, where an incident electron tunnels via a virtual state in the superconductor. The nonlocal conductance, defined in a three-terminal device as the current response in one normal-metal terminal to a voltage bias between the other normal metal and the superconductor, is determined by contributions from both crossed Andreev reflection and electron cotunneling. Crossed Andreev reflection and electron cotunneling give opposite contributions to the nonlocal conductance. In this way, crossed Andreev reflection competes with electron cotunneling. The realization of a system where crossed Andreev reflection can be observed, has been the aim of both experimental ${ }^{3,4}$ and theoretical ${ }^{5-9}$ work lately. This interest is due to the fact that crossed Andreev reflection is an inherently mesoscopic phenomenon, with the prospect of creating entangled electrons. ${ }^{10-12}$

The nonlocal conductance of a device where two normal conductors are tunnel coupled to a bulk superconductor was calculated in second order perturbation theory for quantum tunneling ${ }^{13}$ in Ref. 5. The conductance originating from crossed Andreev reflection was predicted to exactly cancel the conductance due to electron cotunneling. Subsequently, disorder ${ }^{14}$ and higher order quantum interference effects ${ }^{15,16}$ have been incorporated into this approach, and the noise and cross correlations have been considered. ${ }^{17}$ Ferromagnetic contacts were also considered in Ref. 5. Crossed Andreev reflection is favored in an antiparallel configuration of the magnetizations, since Cooper pairs in singlet superconductors consist of two electrons with opposite spins. Electron cotunneling is favored in a parallel configuration.

The predicted dependence of the nonlocal conductance on the magnetization configuration was observed experimentally in a hybrid superconducting-ferromagnetic device. ${ }^{3}$ Subsequently, a bias dependent nonlocal conductance was observed in a more complicated geometry with only normalmetal contacts to the superconductor. ${ }^{4}$ For bias voltages corresponding to energies below the Thouless energy associated with the distance between the two normal terminals, a nonlocal signal with sign corresponding to electron cotunneling was seen. Thus, in contrast to the results of Refs. 5 and 14, experiments showed a finite nonlocal conductance at low bias. Additionally, the sign of the nonlocal signal in Ref. 4 changes when the bias voltage exceeds the Thouless energy, and this was interpreted as a consequence of crossed Andreev reflection dominating the nonlocal signal. These experimental findings are currently not understood.

In previous theoretical works, it is assumed that superconducting properties, e.g., the magnitude of the gap, are not modified by the presence of the contacts. This assumption is valid as long as the coupling between the normal or ferromagnetic conductor and the superconductor is weak or has a small cross section compared to the superconducting coherence length. None of the mentioned theoretical works describe a dependence in the conductances on the Thouless energy.

The circuit theory of mesoscopic transport ${ }^{18,19}$ is a suitable framework to understand transport properties of mesoscopic small normal-metal-superconducting systems. Circuit theory is a discretization of the quasiclassical theory of superconductivity, ${ }^{20}$ and can treat nonequilibrium effects and dephasing. A circuit is modeled as a network of cavities, connectors, and terminals-similar to the way we understand classical electric circuits. Terminals are voltage probes in lo- 
cal thermodynamic equilibrium, whereas cavities can be driven out of equilibrium. Cavities and terminals may be normal metals or superconductors. The connectors can represent physical interfaces between cavities and terminals or model diffusion. Connectors representing interfaces are described by their sets of transmission probabilities. "Kirchhoff's rules" determine the matrix Green's functions (potentials) of the cavities and the matrix currents through the connectors. The matrix currents describe not only the flow of charge and energy, but, e.g., also the flow of quasiparticle correlation. Circuit theory has been applied successfully to explain various phenomena in superconductor and normalmetal-ferromagnet hybrid structures, like the proximity effect, ${ }^{18}$ multiple Andreev reflections, ${ }^{21}$ spin transport, ${ }^{22,23}$ and unconventional superconductivity. ${ }^{24}$ Circuit theory has also proved to be a successful approach to calculate the full counting statistics of hybrid structures. ${ }^{25-28}$ A circuit theory of magnetoelectronics has been developed for hybrid systems of ferromagnets and normal metals; see Ref. 29 and references therein.

We apply circuit theory to calculate the nonlocal conductance of a three-terminal device with contacts to one superconducting and two normal-metal terminals. We substantially generalize previous theoretical approaches by computing analytically the nonlocal conductance with general connectors ranging from, e.g., ballistic point contacts via diffusive contacts to tunnel junctions. We take the proximity effect into account, in which superconducting correlations affect the spectral properties of a normal metal. We also take dephasing into account, where the dwell time gives rise to an effective Thouless energy. We consider low bias transport so that the relevant energies are much smaller than the superconducting gap. The model we consider has a simple and generic geometrical structure. We do not consider Josephson effects. We recover several aspects seen in experiments. ${ }^{4}$ Crossed Andreev reflection and electron cotunneling do not cancel each other. However, in the limiting case of strong coupling between the device and the superconducting terminal, our results agree with previous theoretical work, and the nonlocal conductance vanishes. The differential conductances depend on the effective Thouless energy. A dependence on the Thouless energy has been observed experimentally. ${ }^{4}$ However, we do not find an agreement with the sign of the measured nonlocal conductance above the Thouless energy for the simple model we study.

The paper is organized in the following way. In Sec. II we give an overview of our model and the circuit components. In Sec. III we present the mathematical description and calculation that determine the conductances associated with the various transport processes in the system. We show numerical results for some experimentally relevant systems in Sec. IV. Finally, we give our conclusions in Sec. V.

\section{MODEL}

We consider a three-terminal system where one superconducting terminal and two normal-metal terminals are connected to a small normal-metal cavity. We assume that the cavity is large enough that charging effects can be neglected,

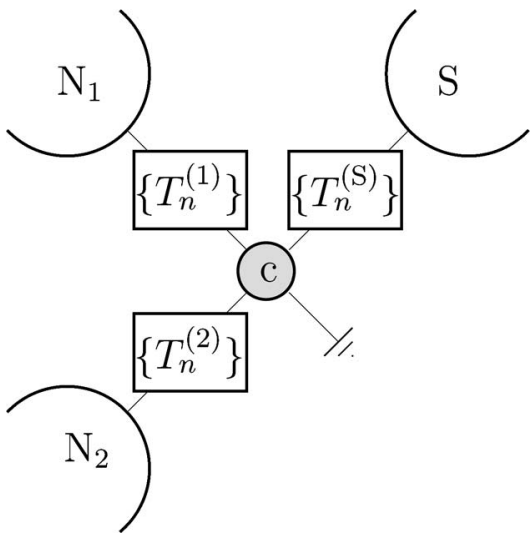

FIG. 1. Our circuit theory model. A normal-metal chaotic cavity (c) is connected to one superconducting (S) and two normal-metal terminals $\left(\mathrm{N}_{1}\right.$ and $\left.\mathrm{N}_{2}\right)$. The three connectors are described by their sets of transmission probabilities. A coupling to ground represents the "leakage of coherence" (see text).

and that the Green's functions are isotropic due to chaotic scattering. A physical realization of the chaotic cavity could be a small piece of diffusive metal, embedded in a circuit by, e.g., tunneling contacts to the terminals. The assumptions on the chaotic cavity are quite general and can also be satisfied for, e.g., a quantum dot with ballistic point contacts, if the conductance of the contacts is much less than the Sharvin estimation of the cavity conductance. ${ }^{19}$ The circuit theory representation of our model is shown in Fig. 1. The normal terminals $\mathrm{N}_{1}$ and $\mathrm{N}_{2}$, and the superconducting terminal $\mathrm{S}$ are connected to the chaotic cavity $\mathrm{c}$ through general connectors represented by their sets of transmission probabilities $\left\{T_{n}^{(i)}\right\}$ where $i=1,2, \mathrm{~S}$, and the index $n$ numbers the conductance channels. These connectors can represent anything from ballistic point contacts to tunnel junctions. ${ }^{22}$ For a ballistic connector all transmission eigenvalues are equal to 1 for the propagating channels and 0 otherwise. For a tunnel junction, all transmission probabilities are small. Dephasing is represented in the circuit diagram by a coupling to ground, although no energy or charge current can flow to this terminal. The dephasing will be discussed in more detail in Sec. III. Our model has a generic geometrical structure and will capture the physics of crossed Andreev reflection and electron cotunneling for a wide range of systems.

Let us now identify the various transport processes in the system. We expect the following contributions to the current: Electron cotunneling (EC) between terminals $\mathrm{N}_{1}$ and $\mathrm{N}_{2}$, direct Andreev (DA) reflection between the superconductor and either normal terminal $\mathrm{N}_{1}$ or $\mathrm{N}_{2}$, and crossed Andreev (CA) reflection between the superconductor and both normal metal terminals $\mathrm{N}_{1}$ and $\mathrm{N}_{2}$. In direct Andreev reflection, an injected particle from one terminal gives rise to a reflected hole in the same terminal, whereas in crossed Andreev reflection an injected particle from terminal $\mathrm{N}_{2}\left(\mathrm{~N}_{1}\right)$ gives rise to a reflected hole in terminal $\mathrm{N}_{1}\left(\mathrm{~N}_{2}\right)$. These processes are illustrated in Fig. 2.

Semiclassical probability arguments show that the spectral charge current in the connector between $N_{1}$ and $c$ at energy $E$ has the following structure: ${ }^{22}$ 


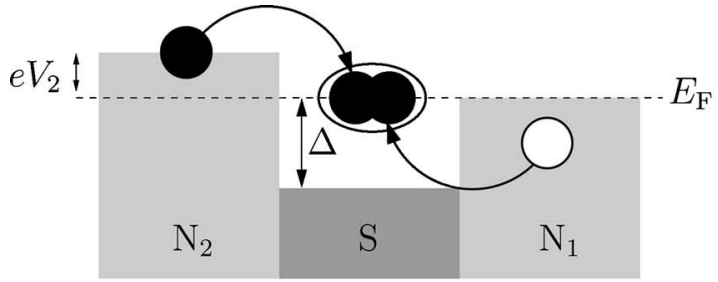

(a)

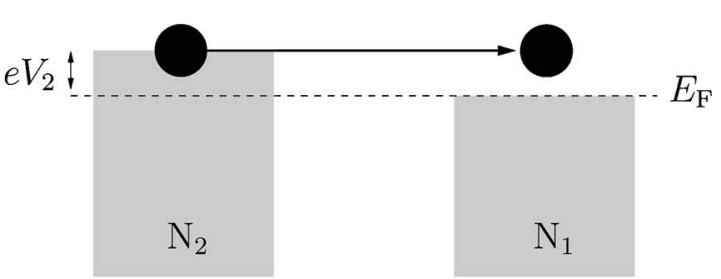

(b)

FIG. 2. Transport processes in the three-terminal device. (a) Crossed Andreev reflection: A particle from $\mathrm{N}_{2}$ with energy $e V_{2}$ and a particle from $\mathrm{N}_{1}$ with energy $-e V_{2}$ form a Cooper pair in $\mathrm{S}$. (b) Electron cotunneling: A particle from $\mathrm{N}_{2}$ at energy $e V_{2}$ tunnels through the cavity $\mathrm{c}$ into $\mathrm{N}_{1}$. The density of states in the cavity $\mathrm{c}$ is suppressed due to the proximity effect from the superconducting terminal.

$$
\begin{aligned}
I_{1}(E)= & \frac{G_{\mathrm{EC}}(E)}{e}\left[f_{2}(E)-f_{1}(E)\right] \\
& +2 \frac{G_{\mathrm{DA}}(E)}{e}\left[1-f_{1}(E)-f_{1}(-E)\right] \\
& +\frac{G_{\mathrm{CA}}(E)}{e}\left[1-f_{1}(E)-f_{2}(-E)\right],
\end{aligned}
$$

where $f_{i}( \pm E)$ denote the Fermi-Dirac distribution functions in normal terminal $i$ at energy $\pm E$. The energy dependent conductances $G(E)$ are even functions of energy. Andreev reflection couples an electron with energy $E$ in terminal $\mathrm{N}_{1}$ to an electron with energy $-E$ in either terminal $\mathrm{N}_{1}$ (DA) or $\mathrm{N}_{2}$ (CA). The factor 2 for direct Andreev reflection takes into account that two charges are transmitted in this process. We divide currents and distribution functions into even and odd parts with respect to energy. The even part contributes to spectral charge current, and the odd part contributes to spectral energy current. We therefore construct $I_{\mathrm{T}, 1}(E) \equiv\left[I_{1}(E)\right.$ $\left.+I_{1}(-E)\right] / 2$ :

$$
\begin{aligned}
I_{\mathrm{T}, 1}(E)= & \frac{1}{e}\left(\frac{1}{2} G_{\mathrm{EC}}+\frac{1}{2} G_{\mathrm{CA}}+2 G_{\mathrm{DA}}\right) h_{\mathrm{T}, 1} \\
& -\frac{1}{2 e}\left(G_{\mathrm{EC}}-G_{\mathrm{CA}}\right) h_{\mathrm{T}, 2},
\end{aligned}
$$

where $h_{\mathrm{T}, i}$ are the reservoir distribution functions determined by Fermi-Dirac functions. ${ }^{40}$ The total charge current is found by integrating over all energies $I_{\text {charge, } 1}=\int d E I_{\mathrm{T}, 1}$. We define the nonlocal differential conductance as the current response in one normal terminal to a voltage between the other normal terminal and the superconductor. Using Eq. (2), this quantity becomes

$$
\frac{\partial I_{\text {charge }, 1}}{\partial V_{2}}=-\int d E\left[G_{\mathrm{EC}}(E)-G_{\mathrm{CA}}(E)\right] \frac{\partial f\left(E-e V_{2}\right)}{\partial E},
$$

where $V_{2}$ is the voltage in terminal $\mathrm{N}_{2}$. At low temperature, the derivative of the Fermi function gives a $\delta$-function at energy $e V_{2}$. The integral then gives $\partial I_{\text {charge, } 1} / \partial V_{2}$ $=G_{\mathrm{EC}}\left(e V_{2}\right)-G_{\mathrm{CA}}\left(e V_{2}\right)$, thus the nonlocal differential conductance is determined by the difference of the cotunneling conductance and the crossed Andreev reflection conductance for quasiparticles at energy $e V_{2}$. Consequently, a measurement of the nonlocal conductance does not uniquely determine the conductance for both processes.

In Sec. III we show that according to the circuit theory, $G_{\mathrm{EC}}-G_{\mathrm{CA}}$ is positive at all energies for the generic network considered. This means that the conductance resulting from electron cotunneling is larger than the conductance resulting from crossed Andreev reflection, and thus the nonlocal differential conductance is always positive.

The odd part of the current in Eq. (1) with respect to energy contributes to energy transport. Direct Andreev reflection does not contribute since two particles of energies $E$ and $-E$ are transmitted. The spectral energy current in the connector between $\mathrm{N}_{1}$ and $\mathrm{c}$ has the following structure: ${ }^{40}$

$$
I_{\mathrm{L}, 1}(E) \equiv \frac{I_{1}(E)-I_{1}(-E)}{2}=\frac{1}{2 e}\left(G_{\mathrm{EC}}+G_{\mathrm{CA}}\right)\left(h_{\mathrm{L}, 1}-h_{\mathrm{L}, 2}\right) .
$$

The total energy current is obtained from the spectral energy current $I_{\mathrm{L}, 1}$ by $I_{\text {energy, } 1}=\int d E E I_{\mathrm{L}, 1} / e$. The energy current into the terminal is related by the heat capacity to the rate of change of the temperature. Thus a nonlocal differential conductance for energy transport could in principle be measured by considering the heat flow into terminal $\mathrm{N}_{1}$. We define the nonlocal differential conductance for energy transport as the energy current response in one terminal to a voltage between the other normal terminal and the superconductor. From Eq. (4) this quantity becomes

$$
\frac{\partial I_{\text {energy }, 1}}{\partial V_{2}}=-\int d E \frac{E}{e}\left[G_{\mathrm{EC}}(E)+G_{\mathrm{CA}}(E)\right] \frac{\partial f\left(E-e V_{2}\right)}{\partial E} .
$$

At low temperatures, this gives $\partial I_{\text {energy, } 1} / \partial V_{2}=V_{2}\left[G_{\mathrm{EC}}\left(e V_{2}\right)\right.$ $\left.+G_{\mathrm{CA}}\left(e V_{2}\right)\right]$; thus the nonlocal differential conductance for energy transport is determined by the sum of the cotunneling conductance and the crossed Andreev reflection conductance for quasiparticles at energy $e V_{2}$.

The discussion above shows that the conductance of electron cotunneling and crossed Andreev reflection can be determined independently from two experimental quantities. Measurements of the nonlocal differential conductance for both charge and energy transport determine the difference and sum of $G_{\mathrm{EC}}$ and $G_{\mathrm{CA}}$, respectively. Thus the conductances of electron cotunneling and crossed Andreev reflec- 
tion are experimentally accessible and can be compared to results from theoretical models.

\section{CIRCUIT THEORY}

The rules of circuit theory allows calculation of the cavity Green's functions in a network when the terminal Green's functions and structure of the connectors are determined. The terminals are characterized by known quasiclassical equilibrium matrix Green's functions $\check{G}_{i}$ in Nambu-Keldysh space. ${ }^{30}$ The Green's functions depend on quasiparticle energy, and terminal temperature and chemical potential. The cavity Green's function in our model is denoted $\check{G}_{\mathrm{c}}$. The Green's functions are $4 \times 4$ matrices including $2 \times 2$ Keldysh space and $2 \times 2$ Nambu space. For an explanation of our standard matrix notation, see the Appendix. The general expression for the matrix current through the connector between terminal $i$ and the cavity is ${ }^{19}$

$$
\check{I}_{i}=-2 \frac{e^{2}}{\pi \hbar} \sum_{n} T_{n}^{(i)}\left[\check{p}_{n}^{(i)} \check{G}_{i}, \check{G}_{c}\right],
$$

where

$$
\check{p}_{n}^{(i)}=\frac{1}{4+T_{n}^{(i)}\left(\left\{\check{G}_{i}, \check{G}_{\mathrm{c}}\right\}-2\right)} .
$$

$\check{p}_{n}^{(i)}$ commutes with the Green's functions in Eq. (6) since it can be expanded in anticommutators of $\check{G}_{i}$ and $\check{G}_{c} \cdot{ }^{27}$ The matrix inversion in Eq. (7) can be performed analytically in Keldysh space due to the symmetries of these matrices. The spectral charge current can be obtained from the expression for the matrix current as $I_{\mathrm{T}, i}=\operatorname{Tr}\left\{\hat{\sigma}_{3} \hat{I}_{i}^{(\mathrm{K})}\right\} / 8 e$, and the spectral energy current as $I_{\mathrm{L}, i}=\operatorname{Tr}\left\{\hat{I}_{i}^{(\mathrm{K})}\right\} / 8 e$. The K superscript denotes the Keldysh matrix block of the current.

Correlation between quasiparticles with opposite excitation energy from the Fermi surface is induced in the cavity due to Andreev scattering at the superconducting terminal. Cooper pairs transferred from the superconductor into the cavity give rise to an electron with excitation energy $E$ and a hole with excitation energy $-E$. The electron and hole quantum wave functions are initially in phase, but a relative phase will arise due to a small mismatch of the wave vectors. ${ }^{19}$ Their wave vectors are $k=k_{\mathrm{F}} \sqrt{1 \pm E / E_{\mathrm{F}}}$ where $k_{\mathrm{F}}$ is the Fermi momentum and $E_{\mathrm{F}}$ the Fermi energy. The relevant transport energy scale is the maximum of the temperature $k_{\mathrm{B}} T$ and bias voltage $e V$. The phase difference between the electron and the hole becomes $\Delta \phi \sim 2 E \tau / \hbar \approx 2 \max \left(e V, k_{\mathrm{B}} T\right) \tau / \hbar$, where the dwell time in the cavity is $\tau$. The dwell time will be discussed in the next paragraph. We denote by $E_{\mathrm{Th}}=\hbar /(2 \tau)$ the effective Thouless energy of the cavity. Let us consider the regime of negligible dephasing, characterized by $\max \left(e V, k_{\mathrm{B}} T\right) \ll E_{\mathrm{Th}}$. The presence of a superconducting terminal leads to prevailing electron-hole correlations since $\Delta \phi \sim \max \left(e V, k_{\mathrm{B}} T\right) / E_{\mathrm{Th}} \ll 1$. In the regime of complete dephasing, on the other hand, $\max \left(e V, k_{\mathrm{B}} T\right) \gg E_{\mathrm{Th}}$, and initial many-particle phase correlation is lost since $\Delta \phi$ is finite and can only be described statistically. Thus the induced su- perconducting correlations due to Andreev scattering are lost, and the wave functions of the quasiparticles in the cavity are not in phase. This dephasing effect is described in circuit theory by an additional terminal for "leakage of coherence."19 Note that no charge or energy flows into this terminal. Circuit theory emerges from a discretization of the Usadel equation, and the dephasing term stems from the energy term in this differential equation. The expression for the matrix current due to dephasing is ${ }^{19}$

$$
\check{I}_{\mathrm{D}}=i e^{2} \nu_{0} V_{\mathrm{c}} E\left[\hat{\sigma}_{3} \overline{\mathbb{1}}, \check{G}_{\mathrm{c}}\right] / \hbar
$$

where $\nu_{0}$ is the density of states in the normal state and $V_{\mathrm{c}}$ is the volume of the cavity.

Let us now discuss the dwell time $\tau$ defined above. The dwell time can be expressed as $\tau=e^{2} \nu_{0} V_{\mathrm{c}} R_{\text {total }}$. $R_{\text {total }}$ is the total resistance to escape from the system, and it includes contributions from the contacts and diffusion. Diffusion is modeled by representing the diffusive region as a network of cavities connected by tunnel-like conductors with resistance times area $\widetilde{r}=\rho d$. Here $\rho$ is the resistivity and $d$ the lattice size in the discretized network. ${ }^{19}$ These connectors contribute to $R_{\text {total }}$. When diffusion is the dominating contribution to $R_{\text {total }}$, the definition of the effective Thouless energy gives $E_{\mathrm{Th}}=\hbar D /\left(2 L^{2}\right)$ in agreement with the continuum theory from which the circuit theory is derived. $D$ is the diffusion constant and $L$ the typical length between contacts. $E_{\mathrm{Th}}$ is the relevant energy scale for the proximity effect in diffusive systems with negligible contact resistances. ${ }^{31}$ In this paper, however, we will consider the opposite limit that $R_{\text {total }}$ is dominated by the contact resistances. Spatial variation of the Green's function inside the system is neglected, and we may discretize with only one cavity. The effective Thouless energy is in this case $E_{\mathrm{Th}}=\hbar /\left(2 e^{2} \nu_{0} \widetilde{R} L\right)$, where $\widetilde{R}$ is the sum of the interface resistances in parallel times area. The contact resistances induce an energy scale for dephasing similar to systems where diffusion is the dominant contribution to $R_{\text {total }}$. In Sec. IV we show in numerical calculations that the effective Thouless energy is the relevant energy scale for the proximity effect in the cavity.

We assume that inelastic processes in the cavity can be neglected since the characteristic time for inelastic interaction is assumed to be much larger than the dwell time. The cavity Green's function is determined by demanding matrix current conservation at each energy. The sum of all matrix currents flowing into the cavity should vanish,

$$
\left[-\frac{2 e^{2}}{\pi \hbar} \sum_{\substack{i=1,2, \mathrm{~S} \\ n}} T_{n}^{(i)} \check{p}_{n}^{(i)} \check{G}_{i}+i \frac{e^{2}}{\hbar} \nu_{0} V_{\mathrm{c}} E \hat{\sigma}_{3} \overline{\mathbb{1}}, \check{G}_{\mathrm{c}}\right]=0
$$

This equation determines the Green's function on the cavity, $\check{G}_{\text {c }}$. The retarded and advanced components of $\check{G}_{\mathrm{c}}$ can be parametrized in terms of one complex function $\theta(E)$ as $\hat{G}_{\mathrm{c}}^{\mathrm{R}}$ $=\hat{\sigma}_{3} \cos (\theta)+\hat{\sigma}_{1} \sin (\theta)$ and $\hat{G}_{\mathrm{c}}^{\mathrm{A}}=-\hat{\sigma}_{3} \cos \left(\theta^{*}\right)+\hat{\sigma}_{1} \sin \left(\theta^{*}\right) .{ }^{32}$ The definition of the Green's function implies that $\operatorname{Re}\{\cos (\theta)\}$ is the normalized, energy-dependent density of states in the cavity, $\nu(E) / \nu_{0}$. 
The Keldysh part of the Green's function is parametrized as $\hat{G}_{\mathrm{c}}^{\mathrm{K}}=\hat{G}_{\mathrm{c}}^{\mathrm{R}} \hat{h}_{\mathrm{c}}-\hat{h}_{\mathrm{c}} \hat{G}_{\mathrm{c}}^{\mathrm{A}}$, where $\hat{h}_{\mathrm{c}}=\hat{1} h_{\mathrm{L}, \mathrm{c}}+\hat{\sigma}_{3} h_{\mathrm{T}, \mathrm{c}}$. The normal terminals have Green's functions $\check{G}_{1(2)}=\hat{\sigma}_{3} \bar{\tau}_{3}+\left(\hat{\sigma}_{3} h_{\mathrm{L}, 1(2)}\right.$ $\left.+\hat{1} h_{\mathrm{T}, 1(2)}\right)\left(\bar{\tau}_{1}+i \bar{\tau}_{2}\right)$, and the Green's function of the superconducting terminal is $\check{G}_{\mathrm{S}}=\hat{\sigma}_{1} \overline{\mathrm{I}}$. We have assumed that any bias voltage $e V \ll \Delta$, where $\Delta$ is the gap of the superconducting terminal. Therefore, the only transport process into S is Andreev reflection since there are no accessible quasiparticle states in this terminal.

The retarded part of matrix current conservation, Eq. (9), gives an equation that determines the pairing angle $\theta$, the retarded "Usadel equation" of the cavity:

$$
\begin{gathered}
\left(i \frac{e^{2}}{\hbar} \nu_{0} V_{\mathrm{c}} E-\frac{e^{2}}{\pi \hbar} \sum_{i=1,2 ; n} \frac{T_{n}^{(i)}}{2+T_{n}^{(i)}[\cos (\theta)-1]}\right) \sin (\theta) \\
+\frac{e^{2}}{\pi \hbar} \sum_{n} \frac{T_{n}^{(\mathrm{S})}}{2+T_{n}^{(\mathrm{S})}[\sin (\theta)-1]} \cos (\theta)=0 .
\end{gathered}
$$

The physical effect on the spectral properties from the various terms can be understood by comparing this equation to the corresponding diffusion equation for a bulk superconductor. ${ }^{33-35}$ This is given in Eq. (II.29b) of Ref. 33 and becomes in our notation ${ }^{41}$

$$
\frac{\hbar D}{2} \frac{\partial^{2} \theta}{\partial x^{2}}+\left(i E-\frac{\hbar}{2 \tau_{E}}\right) \sin \theta+\Delta \cos \theta-\frac{\hbar}{\tau_{\mathrm{sf}}} \sin \theta \cos \theta=0,
$$

where $D$ is the diffusion constant, $\Delta$ the gap to be determined self-consistently, $1 / \tau_{E}$ the inelastic scattering rate, and $1 / \tau_{\mathrm{sf}}$ the spin-flip scattering rate. Comparing this to Eq. (10), we see that the coupling to the superconductor induces superconducting correlations, and that the coupling to the normal terminals gives quasiparticles a finite lifetime. Spin-flip scattering could be included by taking into account magnetic impurities in the cavity. We consider a normal-metal cavity. To describe a superconducting cavity, we would have to include a pairing term in the Hamiltonian. This would result in a term in Eq. (9) with the same structure as the term corresponding to coupling to the superconducting terminal. As long as we do not consider Josephson effects, the effect of superconductivity in the cavity could therefore be included by a quantitative renormalization of the coupling strength to $\mathrm{S}$, which is straightforward.

In the regime of complete dephasing, the term proportional to $\sin (\theta)$ dominates in Eq. (10) because of the large factor $E / E_{\mathrm{Th}}$. The solution in this limit is $\theta=0$, which means that there are no electron-hole correlations in the cavity.

Let us now consider the Keldysh part of Eq. (9). We take the trace of this matrix block after first multiplying it by $\hat{\sigma}_{3}$. The resulting equation determines the distribution function $h_{\mathrm{T}, \mathrm{c}}$ in the cavity,

$$
G_{\mathrm{T}, 1}\left(h_{\mathrm{T}, 1}-h_{\mathrm{T}, \mathrm{c}}\right)+G_{\mathrm{T}, 2}\left(h_{\mathrm{T}, 2}-h_{\mathrm{T}, \mathrm{c}}\right)+G_{\mathrm{T}, \mathrm{S}}\left(0-h_{\mathrm{T}, \mathrm{c}}\right)=0 .
$$

This implies charge conservation at each energy, with effective energy-dependent conductances $G_{\mathrm{T}, i}$ between the cavity and terminals $\mathrm{N}_{1}, \mathrm{~N}_{2}$, and $\mathrm{S}$. The zero in the term $G_{\mathrm{T}, \mathrm{S}}(0$
$\left.-h_{\mathrm{T}, \mathrm{c}}\right)$ represents the charge distribution function in the superconductor, $h_{\mathrm{T}, \mathrm{S}}$, which vanishes since the superconductor is grounded. The conductance $G_{\mathrm{T}, \mathrm{S}}$ controls the Andreev reflection rate in the cavity since it is the conductance between the cavity and the superconducting terminal. The effective conductances are given in terms of the pairing angle and the transmission probabilities as

$$
\begin{aligned}
G_{\mathrm{T}, i} & =2 \frac{e^{2}}{\pi \hbar} \sum_{n} T_{n}^{(i)} \frac{\left(2-T_{n}^{(i)}\right) \operatorname{Re}\{\cos (\theta)\}+T_{n}^{(i)} D_{\mathrm{T}}}{\left|2+T_{n}^{(i)}[\cos (\theta)-1]\right|^{2}} \\
G_{\mathrm{T}, \mathrm{S}} & =2 \frac{e^{2}}{\pi \hbar} \sum_{n} T_{n}^{(\mathrm{S})} \frac{\left(2-T_{n}^{(\mathrm{S})}\right) \operatorname{Re}\{\sin (\theta)\}+T_{n}^{(\mathrm{S})} D_{\mathrm{T}}}{\left|2+T_{n}^{(\mathrm{S})}[\sin (\theta)-1]\right|^{2}} .
\end{aligned}
$$

Here $D_{\mathrm{T}}=[\operatorname{Re}\{\cos (\theta)\}]^{2}+[\operatorname{Re}\{\sin (\theta)\}]^{2}$ and $i=1,2$. The term proportional to $G_{\mathrm{T}, \mathrm{S}}$ in Eq. (11) describes conversion of quasiparticles in the cavity into condensate in the superconducting terminal. There is an analogous term in the Boltzmann equation for a continuum superconductor, ${ }^{35,36}$ which describes conversion between quasiparticles and superconducting condensate over the coherence length. This analogous term has a similar dependence on $\theta$. The rate of this conversion is controlled by $\Delta$ in the continuum case, and by $G_{\mathrm{T}, \mathrm{S}}$ in the cavity of our discretized theory.

In the regime of complete dephasing $\theta=0$, the conductances to the normal terminals $G_{\mathrm{T}, i}$ coincide with the Landauer-Büttiker formula. $G_{\mathrm{T}, \mathrm{S}}=2 e^{2} \Sigma_{n}\left(T_{n}^{(S)}\right)^{2} /[\pi \hbar(2$ $\left.\left.-T_{n}^{(S)}\right)^{2}\right]$ corresponds to the Andreev conductance of an N-S interface in a diffusive system, calculated by Beenakker. ${ }^{37}$ Thus the distribution function $h_{\mathrm{T}, \mathrm{c}}$ can be determined in the regime of complete dephasing from well-known results by demanding charge current conservation.

In the tunnel barrier limit, all transmission probabilities are small, and we can expand to first order in $T_{n}^{(i)}$ in Eqs. (12). We define $g_{i}=e^{2} \Sigma_{n} T_{n}^{(i)} /(\pi \hbar)$ for $i=1,2, \mathrm{~S}$. For the normal terminals $(i=1,2)$ we find $G_{\mathrm{T}, i}=g_{i} \operatorname{Re}\{\cos (\theta)\}$. $\operatorname{Re}\{\cos (\theta)\}$ gives the normalized density of states in the cavity which is under the influence of the proximity effect. The tunnel conductance to the superconductor becomes $G_{\mathrm{T}, \mathrm{S}}$ $=g_{\mathrm{S}} \operatorname{Re}\{\sin (\theta)\}$, which vanishes when there is complete dephasing, $\theta=0$. This is expected since the Andreev conductance of a tunnel barrier between incoherent normal and superconducting terminals vanishes. ${ }^{38}$

The trace of the Keldysh block of Eq. (9) gives an equation that determines the distribution function $h_{\mathrm{L}, \mathrm{c}}$ in the cavity,

$$
G_{\mathrm{L}, 1}\left(h_{\mathrm{L}, 1}-h_{\mathrm{L}, \mathrm{c}}\right)+G_{\mathrm{L}, 2}\left(h_{\mathrm{L}, 2}-h_{\mathrm{L}, \mathrm{c}}\right)=0 .
$$

This is energy conservation at each energy, with effective energy-dependent conductances for energy transport $G_{\mathrm{L}, i}$. No energy current can flow through the contact between the cavity and the superconducting terminal since no net energy is transferred into $S$ by Andreev reflection. Our calculation is restricted to $E \ll \Delta$, but in the general case a quasiparticle current which carries energy can flow into the superconducting terminal for $E>\Delta$. The effective conductances for en- 
ergy transport are given in terms of the pairing angle and the transmission probabilities as

$$
G_{\mathrm{L}, i}=2 \frac{e^{2}}{\pi \hbar} \sum_{n} T_{n}^{(i)} \frac{\left(2-T_{n}^{(i)}\right) \operatorname{Re}\{\cos (\theta)\}+T_{n}^{(i)} D_{\mathrm{L}}}{\left|2+T_{n}^{(i)}[\cos (\theta)-1]\right|^{2}} .
$$

Here $D_{\mathrm{L}}=[\operatorname{Re}\{\cos (\theta)\}]^{2}-[\operatorname{Im}\{\sin (\theta)\}]^{2}$ and $i=1,2$.

In the tunnel barrier limit we find that $G_{\mathrm{L}, i}=G_{\mathrm{T}, i}$ $=g_{i} \operatorname{Re}\{\cos (\theta)\}$ for $i=1,2$, which means that the effective conductances for energy transport and charge transport into the normal terminals are equal. The conductances correspond to the usual quasiparticle tunnel conductance in this case.

Equations (10), (11), and (13) determine the Green's function in the cavity. The charge and energy currents $I_{\mathrm{T}, i}$ and $I_{\mathrm{L}, i}$ between terminal $i$ and the cavity can be calculated once $\breve{G}_{\mathrm{c}}$ is known. Comparison of Eqs. (2) and (4) with the expressions for $I_{\mathrm{T}, 1}$ and $I_{\mathrm{L}, 1}$ obtained from circuit theory, allows us to determine the conductances associated with the various transport processes:

$$
\begin{aligned}
& G_{\mathrm{DA}}(E)=\frac{1}{4}\left(\frac{G_{\mathrm{T}, 1}\left(G_{\mathrm{T}, 2}+G_{\mathrm{T}, \mathrm{S}}\right)}{G_{\mathrm{T}, 1}+G_{\mathrm{T}, 2}+G_{\mathrm{T}, \mathrm{S}}}-\frac{G_{\mathrm{L}, 1} G_{\mathrm{L}, 2}}{G_{\mathrm{L}, 1}+G_{\mathrm{L}, 2}}\right), \\
& G_{\mathrm{CA}}^{\mathrm{EC}}(E)=\frac{1}{2}\left(\frac{G_{\mathrm{L}, 1} G_{\mathrm{L}, 2}}{G_{\mathrm{L}, 1}+G_{\mathrm{L}, 2}} \pm \frac{G_{\mathrm{T}, 1} G_{\mathrm{T}, 2}}{G_{\mathrm{T}, 1}+G_{\mathrm{T}, 2}+G_{\mathrm{T}, \mathrm{S}}}\right) .
\end{aligned}
$$

These formulas are the main result of our calculation. Equation (15b) shows that $G_{\mathrm{EC}}-G_{\mathrm{CA}}$ is positive. Thus the nonlocal conductance $\partial I_{1} / \partial V_{2}$ is positive. In the limit where the coupling to the superconducting terminal vanishes, i.e., all $T_{n}^{(\mathrm{S})} \rightarrow 0$, only $G_{\mathrm{EC}}$ remains nonzero and the conductance agrees with the result for a normal double-barrier system. If the conductance to one of the normal terminals vanishes, i.e., all $T_{n}^{(i)} \rightarrow 0$ for, e.g., $i=2$, only $G_{\mathrm{DA}}$ is nonzero. When the coupling between the superconducting terminal and the cavity is very strong $G_{\mathrm{T}, \mathrm{S}} \gg G_{\mathrm{T}, 1}, G_{\mathrm{T}, 2}$, we recover the result of Ref. 5 that $G_{\mathrm{EC}}=G_{\mathrm{CA}}$, which means that the nonlocal conductance vanishes since transport by electron cotunneling is exactly canceled by crossed Andreev reflection.

To describe a device where spatial variation in a bulk region is important, a model with several cavities connected in a network is required. The connectors between cavities represent the intrinsic resistance due to diffusion, and contribute to $R_{\text {total }}$ and thus to the effective Thouless energy $E_{\mathrm{Th}}=\hbar /\left(2 e^{2} \nu_{0} V_{\mathrm{c}} R_{\text {total }}\right)$. However, as long as there are no Josephson currents in the network, the symmetry $G_{\mathrm{EC}(\mathrm{CA})}=\alpha$ $+(-) \beta$ of Eq. (15b) persists. Here $\alpha$ and $\beta$ are positive numbers. This follows since the currents flowing out of the normal terminals given by circuit theory can be written $I_{\mathrm{L}, i}(E)$ $=C_{\mathrm{L}}^{(i)}\left(h_{\mathrm{L}, 1}-h_{\mathrm{L}, 2}\right)$ and $I_{\mathrm{T}, i}(E)=C_{\mathrm{T} 1}^{(i)} h_{\mathrm{T}, 1}-C_{\mathrm{T} 2}^{(i)} h_{\mathrm{T}, 2}$ where $C_{\mathrm{L}}^{(i)}$, $C_{\mathrm{T} 1}^{(i)}$, and $C_{\mathrm{T} 2}^{(i)}$ are coefficients. Comparing these expressions to Eqs. (2) and (4), we see, e.g., that $G_{\mathrm{EC}}-G_{\mathrm{CA}}=2 e C_{\mathrm{T} 2}^{(i)}>0$ for contact $i$, regardless of the internal structure of the network of cavities. Thus the sign of the nonlocal conductance is not affected by diffusion or network geometry. We interpret this result as the consequence of a symmetry between the crossed Andreev reflection and electron cotunneling. Both processes involve the transfer of quasiparticles through the contacts to the normal metals and the network between them, but the crossed Andreev reflection also involves Andreev reflection at the interface to the superconducting terminal. Thus the resistance limiting crossed Andreev reflection can at minimum be as small as the resistance for electron cotunneling unless other physical processes affect these quantities. We believe that an explanation of the measurements in Ref. 4 requires additional physical effects not considered here.

\section{A. Analytically solvable limits}

In this section, we give results for two limits where it is possible to solve Eq. (10) analytically and obtain simple expressions for the conductances in Eqs. (15).

\section{The regime of complete dephasing}

When $E \gg E_{\mathrm{Th}}$ there is complete dephasing. In this case the conductances $G_{\mathrm{T}, i}=G_{\mathrm{L}, i}=g_{i}$ for $i=1,2$ agree with the Landauer-Büttiker formula and $G_{\mathrm{T}, \mathrm{S}}$ is Beenakker's result for the conductance of an $\mathrm{N}-\mathrm{S}$ interface in a diffusive system, as noted above. In this limit, the conductances in Eq. (15) become

$$
\begin{gathered}
G_{\mathrm{DA}}=\frac{g_{1}^{2}}{4} \frac{G_{\mathrm{T}, \mathrm{S}}}{\left(g_{1}+g_{2}\right)\left(g_{1}+g_{2}+G_{\mathrm{T}, \mathrm{S}}\right)}, \\
G_{\mathrm{CA}}^{\mathrm{EC}}(E)=\frac{g_{1} g_{2}}{2} \frac{1}{\left(g_{1}+g_{2}\right)\left(g_{1}+g_{2}+G_{\mathrm{T}, \mathrm{S}}\right)} \\
\times \begin{cases}2\left(g_{1}+g_{2}\right)+G_{\mathrm{T}, \mathrm{S}} & \text { for EC, } \\
G_{\mathrm{T}, \mathrm{S}} & \text { for CA. }\end{cases}
\end{gathered}
$$

The currents resulting from these expressions using Eq. (1) can also be calculated from a semiclassical approach by demanding charge conservation in the cavity using the wellknown theory for incoherent N-S transport. The result for $G_{\mathrm{EC}}-G_{\mathrm{CA}}$ was shown in Ref. 7, where also spin polarizing contacts are employed to give a negative nonlocal conductance. Again, in the limit of strong coupling to the superconductor, $g_{i} / G_{\mathrm{T}, \mathrm{S}} \ll 1$, we see that $G_{\mathrm{EC}}=G_{\mathrm{CA}}$ and thus the nonlocal conductance vanishes.

\section{Tunnel barrier limit}

In the tunnel barrier limit, all transmission probabilities are small. We expand to first order in $T_{n}^{(i)}$ in the expressions for the matrix currents. This corresponds to putting $\check{p}_{n}^{(i)}$ $\rightarrow 1 / 4$ in Eq. (6). The resulting Eq. (9) can be solved analytically without resorting to a parametrization of $\check{G}_{\mathrm{c}}{ }^{19}$ The solution is of course equivalent to what we obtain from Eqs. (10), (11), and (13) in the same limit. Let us consider the resulting expressions for the conductances of nonlocal transport in some limits. At zero energy, we obtain

$$
G_{\mathrm{DA}}=\frac{g_{1}^{2}}{4} \frac{g_{\mathrm{S}}^{2}}{\left[\left(g_{1}+g_{2}\right)^{2}+g_{\mathrm{S}}^{2}\right]^{3 / 2}},
$$




$$
\begin{aligned}
G_{\mathrm{EC}} & =\frac{g_{1} g_{2}}{2} \frac{2\left(g_{1}+g_{2}\right)^{2}+g_{\mathrm{S}}^{2}}{\left[\left(g_{1}+g_{2}\right)^{2}+g_{\mathrm{S}}^{2}\right]^{3 / 2}}, \\
G_{\mathrm{CA}} & =\frac{g_{1} g_{2}}{2} \frac{g_{\mathrm{S}}^{2}}{\left[\left(g_{1}+g_{2}\right)^{2}+g_{\mathrm{S}}^{2}\right]^{3 / 2}} .
\end{aligned}
$$

These results correspond to completely phase-coherent transport. The full counting statistics for the same system in this regime has been calculated in Ref. 28. In that paper, it is found that the cross-correlation noise can have both signs in the three-terminal device.

If there is complete dephasing, the cavity spectral properties are like those of a normal metal. This gives $G_{\mathrm{T}, \mathrm{S}}=0$ since the Andreev conductance of a tunnel barrier vanishes for incoherent N-S transport. ${ }^{38}$ Therefore, there are no transport channels into the superconducting terminal and it is effectively isolated from the circuit. Only the conductance for electron cotunneling is nonzero, and we obtain from (17b)

$$
G_{\mathrm{EC}}=\frac{1}{1 / g_{1}+1 / g_{2}},
$$

i.e., addition of the conductances between the cavity and both normal-metal terminals in series. This result corresponds to normal-state tunneling between $\mathrm{N}_{1}$ and $\mathrm{N}_{2}$.

\section{RESULTS}

In an experimental situation, nonlocal transport can be probed in measurements of the voltage or current in terminal $\mathrm{N}_{1}$ resulting from the injection of current through terminal $\mathrm{N}_{2}{ }^{3,4}$ At zero temperature, the nonlocal differential conductance $\partial I_{\text {charge, } 1} / \partial V_{2}$ as a function of $e V_{2} / E_{\mathrm{Th}}$ corresponds to $G_{\mathrm{EC}}-G_{\mathrm{CA}}$ [see Eq. (3)]. This quantity is given by Eqs. (15b) and is always positive within our model. The detailed behavior as a function of $e V_{2}$ depends on the pairing angle of the cavity, $\theta$, which is determined by Eq. (10). This equation can be solved numerically, and determines the spectral properties and thus the nonlocal conductances when the parameters of the model are fixed. We will show results for three systems with different combinations of contacts which can be fabricated with state of the art nanotechnology. The contacts we study are tunnel barriers or metallic contacts. The systems represent various realizations where our circuit theory model applies. We show that the nonlocal conductances are very sensitive to the type of contacts in the system, and have a strong dependence on the Thouless energy. We have also investigated effect of inelastic scattering inside the cavity, and have found no notable qualitative differences on the conductances as compared to the elastic case; thus these results are not shown here.

\section{A. Tunnel barriers}

When all the three contacts are tunnel barriers, the equation for matrix current conservation can be solved analytically as mentioned in Sec. III A 2. The resulting expressions are quite complicated. We expand to first order in the transmission probabilities since $T_{n}^{(i)} \ll 1$ for $i=1,2$, S, and let $g_{i}$

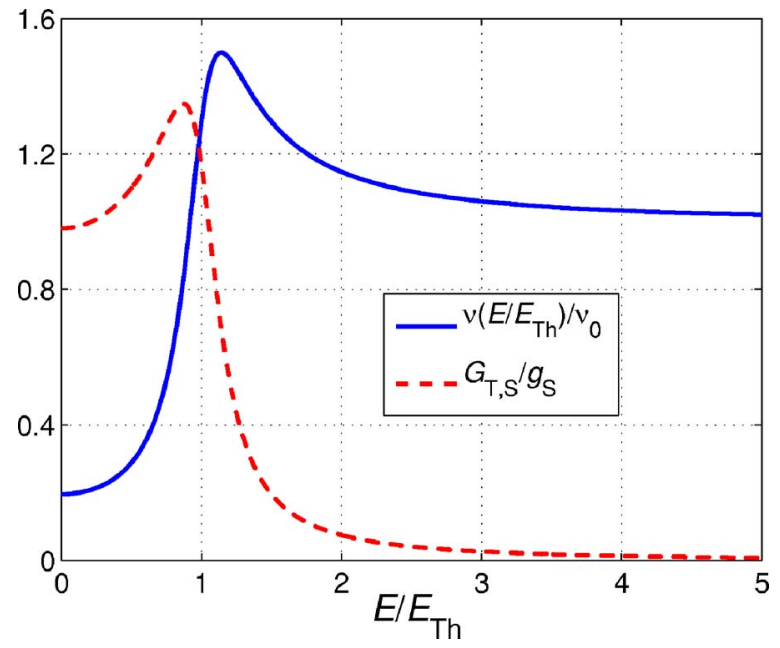

FIG. 3. (Color online) Spectral properties of the cavity when all contacts are of tunnel type with $g_{1} / g_{\mathrm{S}}=g_{2} / g_{\mathrm{S}}=0.1$. Solid line (blue) shows normalized density of states $\nu(E) / \nu_{0}$. Dashed line (red) shows $G_{\mathrm{T}, \mathrm{S}} / g_{\mathrm{S}}$ which is the parameter that determines the Andreev reflection rate of quasiparticles.

denote tunnel barrier conductance in the normal-metal state. Let us first consider a symmetric system, $g_{1} / g_{\mathrm{S}}=g_{2} / g_{\mathrm{S}}=0.1$. We define the Thouless energy in this case as $E_{\mathrm{Th}}$ $=\hbar g_{\mathrm{S}} /\left(2 e^{2} \nu_{0} V_{\mathrm{c}}\right)$. The spectral properties of the cavity are shown in Fig. 3. In this plot we show the normalized density of states in the cavity and the conductance $G_{\mathrm{T}, \mathrm{S}}$ which controls the Andreev reflection rate. From Fig. 3 we see that below the Thouless energy of the cavity, the quasiparticle density of states is suppressed due to electron-hole correlations. This affects all transport processes since they rely on quasiparticles propagating through the cavity. Above the Thouless energy, the density of states approaches the value in the normal state. This is the typical behavior for proximity coupled systems. ${ }^{34}$ The conductance between the cavity and the superconducting terminal, $G_{\mathrm{T}, \mathrm{S}}$, approaches the normalstate value at low energies. At high energies, $G_{\mathrm{T}, \mathrm{S}}$ vanishes, as expected for tunnel barriers in the regime $E<\Delta$ when the proximity effect can be neglected. ${ }^{38}$ This affects the crossed and direct Andreev reflections, which vanish when $G_{\mathrm{T}, \mathrm{S}}$ goes to zero.

A plot of the conductances for electron cotunneling, and crossed and direct Andreev reflections is shown in Fig. 4. The conductances have a rapid increase near $E=E_{\mathrm{Th}}$ related to the energy dependence of the density of states. At high energies $G_{\mathrm{EC}}$ approaches the value for normal double-barrier tunneling, Eq. (18). $G_{\mathrm{CA}}$ and $G_{\mathrm{DA}}$ vanish at high energy. These conductances are determined by an interplay of the density of states and the Andreev reflection rate, and is small when either of these quantities is small. The measurable nonlocal differential conductance $G_{\mathrm{EC}}-G_{\mathrm{CA}}$ is a monotonically increasing function with increasing energy, which starts at a small value and approaches $G_{\mathrm{EC}}$ above the Thouless energy.

Let us now consider the effect of asymmetry between the tunnel barriers to the normal metals. The expressions for the conductances at zero energy, Eqs. (17), show that the direct Andreev conductance of one contact, $G_{\mathrm{DA}}^{(1)}$ for example, is proportional to $g_{1}^{2}$ since two electrons have to tunnel through 


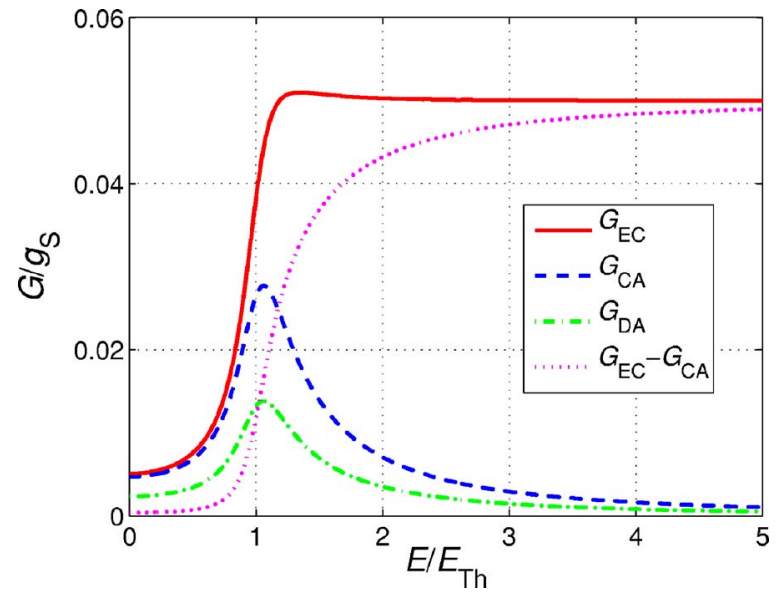

FIG. 4. (Color online) Conductance for the various transport processes through the cavity when all contacts are of tunnel type. The two normal contacts have the same conductance in this plot, $g_{1} / g_{\mathrm{S}}=g_{2} / g_{\mathrm{S}}=0.1$. Solid line (red), $G_{\mathrm{EC}} / g_{\mathrm{S}}$; dashed line (blue), $G_{\mathrm{CA}} / g_{\mathrm{S}}$; dash-dotted line (green), $G_{\mathrm{DA}} / g_{\mathrm{S}}$; and dotted line (purple), $\left(G_{\mathrm{EC}}-G_{\mathrm{CA}}\right) / g_{\mathrm{S}}$.

the connector with conductance $g_{1}$. $G_{\mathrm{DA}}^{(1)}$ is, however, only weakly dependent on $g_{2}$. The same is true for the direct Andreev conductance of connector 2 with $g_{1} \leftrightarrow g_{2}$. The direct Andreev conductances are therefore relatively independent of the asymmetry. On the other hand, nonlocal conductances are proportional to $g_{1} g_{2}$ because a quasiparticle has to tunnel through both connectors. These conductances are sensitive to the asymmetry which we define as $a=g_{1} / g_{2}$. For the conductance measured at terminal $\mathrm{N}_{1}$ we see that $G_{\mathrm{EC}(\mathrm{CA})}^{(1)} / G_{\mathrm{DA}}^{(1)}$ $\propto 1 / a$. Conversely, the conductance measured at terminal $\mathrm{N}_{2}$ gives $G_{\mathrm{EC}(\mathrm{CA})}^{(2)} / G_{\mathrm{DA}}^{(2)} \propto a$. Thus asymmetry suppresses the nonlocal conductance of one contact, and enhances the nonlocal conductance of the other contact. In Fig. 5 we show the conductances for a system where $g_{1} / g_{\mathrm{S}}=0.1$ and $g_{2} / g_{\mathrm{S}}=0.3$.

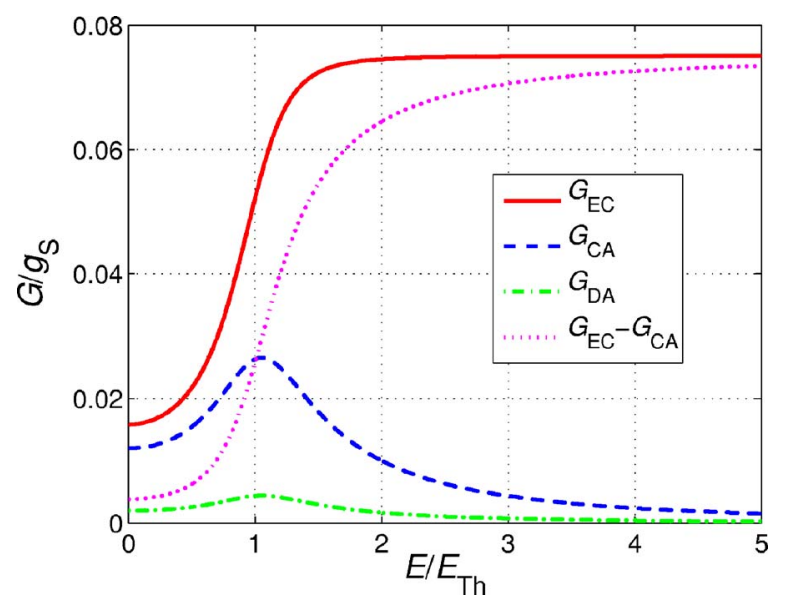

FIG. 5. (Color online) Conductance for the various transport processes through the cavity when all contacts are of tunnel type. Conductances are normalized by $g_{\mathrm{S}}$, and parameter values are $g_{1} / g_{\mathrm{S}}=0.1, g_{2} / g_{\mathrm{S}}=0.3$. Solid line (red), $G_{\mathrm{EC}} / g_{\mathrm{S}}$; dashed line (blue), $G_{\mathrm{CA}} / g_{\mathrm{S}}$; dash-dotted line (green), $G_{\mathrm{DA}} / g_{\mathrm{S}}$; and dotted line (purple), $\left(G_{\mathrm{EC}}-G_{\mathrm{CA}}\right) / g_{\mathrm{S}}$.

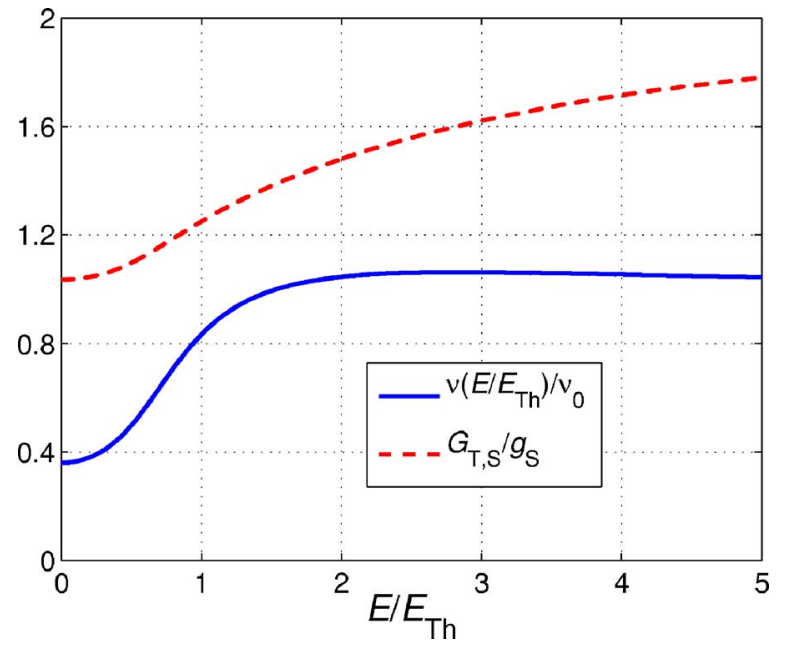

FIG. 6. (Color online) Spectral properties of the cavity when the contact to the superconducting reservoir is metallic with $T_{n}^{(\mathrm{S})}=1$ for all propagating channels and zero otherwise, and the contacts to the normal reservoirs are of tunnel type with $g_{1} / g_{\mathrm{S}}=0.1$ and $g_{2} / g_{\mathrm{S}}$ $=0.3$. Solid line (blue) shows normalized density of states in the cavity, $\nu(E) / \nu_{0}$, and dashed line (red) shows $G_{\mathrm{T}, \mathrm{S}} / g_{\mathrm{S}}$, which is the parameter that determines the Andreev reflection rate of quasiparticles in the cavity.

The asymmetry is now $a=1 / 3$, thus the effect of nonlocal processes is enhanced when we consider the conductances measured at terminal $\mathrm{N}_{1}$. The spectral properties in this case are similar to those of the symmetric system shown in Fig. 3, and are not shown here. Comparing Figs. 4 and 5, we see that the conductances for the crossed and direct Andreev reflections are not as peaked in the asymmetric system as in the symmetric system. We also see that the relative magnitude of the nonlocal conductances to the direct Andreev conductance has increased. In the remainder of the paper, we consider the conductances of contact 1 in asymmetric structures $a=1 / 3$ since we are mostly interested in the conductance resulting from nonlocal processes.

\section{B. Tunnel contacts to $N_{1}$ and $N_{2}$; metallic contact to $S$}

Systems where two normal-metal terminals are connected to a cavity, in which the cavity may itself be part of a larger superconducting structure, can be studied in our model. The contact to the superconductor could in this case be, e.g., diffusive or metallic. In this section, we assume tunnel contacts to the two normal metals, and metallic contact to the superconductor. The metallic contact is described by the transmission probabilities $T_{n}^{(\mathrm{S})}=1$ for all propagating channels and zero otherwise. We choose parameters $g_{1} / g_{\mathrm{S}}=0.1$ and $g_{2} / g_{\mathrm{S}}=0.3$. The Thouless energy is $E_{\mathrm{Th}}=\hbar g_{\mathrm{S}} /\left(2 e^{2} \nu_{0} V_{\mathrm{c}}\right)$. In Fig. 6 we show the spectral properties of the cavity. Below the Thouless energy, the quasiparticle density of states is suppressed. At low energy, $G_{\mathrm{T}, \mathrm{S}}$ approaches the value in the normal state. This is similar to the reentrant behavior in diffusive systems. ${ }^{31,39}$ With increasing energy, the rate of Andreev reflection at the superconducting terminal increases. The value of $G_{\mathrm{T}, \mathrm{S}} / g_{\mathrm{S}}$ reaches its maximum value 2 for 


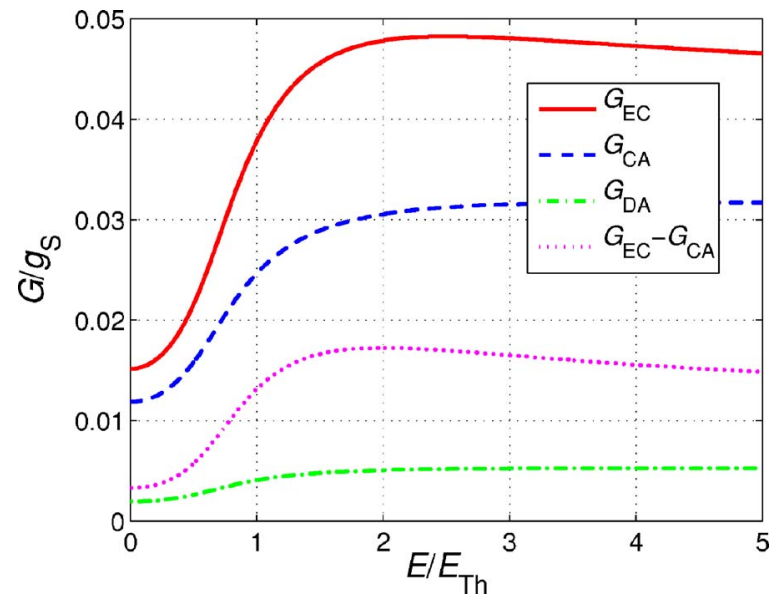

FIG. 7. (Color online) Conductances for the various transport processes when the contact to the superconducting reservoir is metallic with $T_{n}^{(\mathrm{S})}=1$ for all propagating channels and zero otherwise, and the contacts to the normal reservoirs are of tunnel type with $g_{1} / g_{\mathrm{S}}=0.1$ and $g_{2} / g_{\mathrm{S}}=0.3$. Solid line (red), $G_{\mathrm{EC}} / g_{\mathrm{S}}$; dashed line (blue), $G_{\mathrm{CA}} / g_{\mathrm{S}}$; dash-dotted line (green), $G_{\mathrm{DA}} / g_{\mathrm{S}}$; and dotted line (purple), $\left(G_{\mathrm{EC}}-G_{\mathrm{CA}}\right) / g_{\mathrm{S}}$.

$E / E_{\mathrm{Th}} \approx 20$. This result agrees with the Blonder-TinkhamKlapwijk formula for high transmission probabilities, valid when proximity effect is negligible. ${ }^{38}$

The conductances for this system are shown in Fig. 7. All conductances are suppressed below the Thouless energy by the low density of states. Above the Thouless energy, the increasing Andreev reflection rate leads to a suppression of $G_{\mathrm{EC}}$ and an enhancement of $G_{\mathrm{CA}}$. The overall behavior of the nonlocal conductances is determined by the interplay of the dependence on the density of states and the Andreev reflection rate. The two conductances associated with Andreev reflection, $G_{\mathrm{CA}}$ and $G_{\mathrm{DA}}$, reach their maximum at approximately $E / E_{\mathrm{Th}}=4$ and then decrease slowly, reaching their final value at $E / E_{\mathrm{Th}} \approx 20$.

\section{Metallic contacts to $N_{1}$ and $N_{2}$; tunnel contact to $S$}

Let us now consider a system where the normal terminals $\mathrm{N}_{1}$ and $\mathrm{N}_{2}$ are connected to the cavity through metallic contacts described by $T_{n}^{(i)}=1$ for all propagating channels and zero otherwise. The superconducting terminal is connected by a tunnel barrier of conductance $g_{\mathrm{S}}$, and the Thouless energy is $E_{\mathrm{Th}}=\hbar g_{\mathrm{S}} /\left(2 e^{2} \nu_{0} V_{\mathrm{c}}\right)$. The spectral properties of this system are very similar to the tunneling case in Sec. IV A, and we do not show them here. However, the conductances between the normal terminals and the cavity are qualitatively different in the present case. In Fig. 8 we show $G_{\mathrm{T}, 1}$ for both the tunneling case in Sec. IV A, and where the normal terminals are connected by metallic contacts. At energies below the Thouless energy, $G_{\mathrm{T}, 1}$ is in the tunneling case qualitatively similar to the density of states. However, with metallic contacts to the normal reservoirs, $G_{\mathrm{T}, 1}$ is large at zero energy and decreases as the energy increases beyond $E_{\mathrm{Th}}$. At high energy, $G_{\mathrm{T}, 1}$ is equal for the two cases and corresponds to the result in the normal state since $g_{1} / g_{\mathrm{S}}$ is the same for the two curves. $G_{\mathrm{T}, 1}$ for a metallic contact is qualitatively similar to

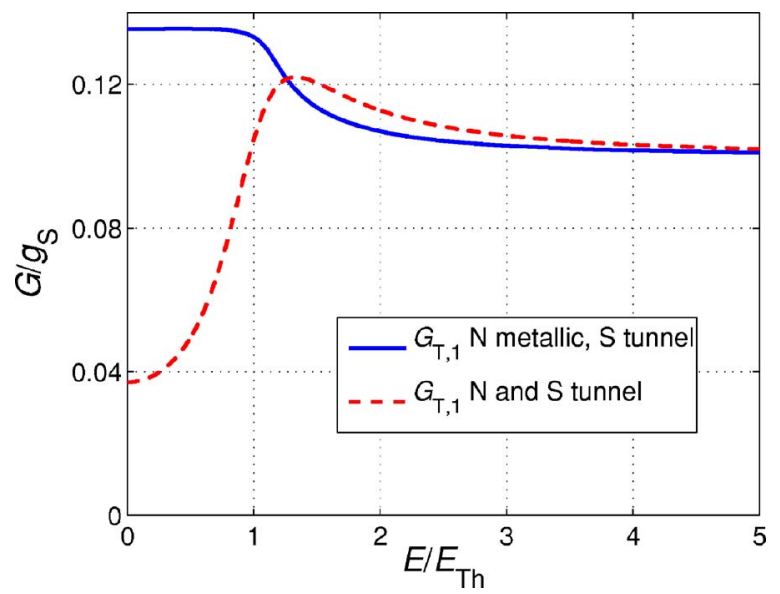

FIG. 8. (Color online) Energy dependence of conductance $G_{\mathrm{T}, 1}$ for the present case of metallic contacts to normal reservoirs and tunnel contact to the superconducting reservoir in solid line (blue), and for the case with only tunnel barriers studied in Sec. IV A in dashed line (red). In both cases we have put the parameters $g_{1} / g_{\mathrm{S}}$ $=0.1$ and $g_{2} / g_{S}=0.3$.

the conductance of a metallic normal-metal-superconductor interface, except that in this case the "superconductor" is the cavity which is under the influence of the proximity effect from $\mathrm{S}$ and the relevant energy scale is $E_{\mathrm{Th}}$ instead of $\Delta$.

The conductances for the present system are shown in Fig. 9. A new feature here is a small dip in $G_{\mathrm{EC}}$ at $E=E_{\mathrm{Th}}$ before the rapid increase above $E_{\mathrm{Th}} . G_{\mathrm{EC}}$ is proportional to $G_{\mathrm{T}, 1}$ and inversely proportional to $G_{\mathrm{T}, \mathrm{S}}$. The dip in $G_{\mathrm{EC}}$ can therefore be understood by the decreasing charge current conductance $\left(G_{\mathrm{T}, 1}\right)$ and the peak in $G_{\mathrm{T}, \mathrm{S}}$ around $E=E_{\mathrm{Th}}$. At higher energy $G_{\mathrm{EC}}$ increases as $G_{\mathrm{T}, \mathrm{S}}$ vanishes. The dip in $G_{\mathrm{EC}}$ leads to a larger dip in $G_{\mathrm{EC}}-G_{\mathrm{CA}}$ since $G_{\mathrm{CA}}$ increases with increasing energy at $E<E_{\mathrm{Th}}$. In the tunneling case of Sec.

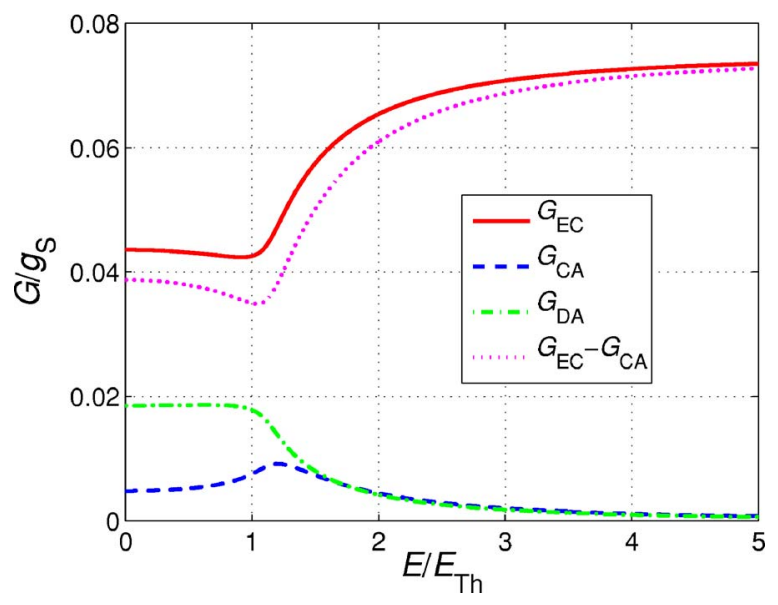

FIG. 9. (Color online) Conductances for the various transport processes when the contacts to the normal reservoirs are of metallic type with $T_{n}^{(i)}=1$ where $i=1,2$ for all propagating channels and zero otherwise, and the contact to the superconducting reservoir is of tunnel type with conductance $g_{\mathrm{S}}$. In this plot $g_{1} / g_{\mathrm{S}}=0.1$ and $g_{2} / g_{\mathrm{S}}=0.3$. Solid line (red), $G_{\mathrm{EC}} / g_{\mathrm{S}}$; dashed line (blue), $G_{\mathrm{CA}} / g_{\mathrm{S}}$; dash-dotted line (green), $G_{\mathrm{DA}} / g_{\mathrm{S}}$; and dotted line (purple), $\left(G_{\mathrm{EC}}\right.$ $\left.-G_{\mathrm{CA}}\right) / g_{\mathrm{S}}$. 
IV A, the increase of the Andreev conductance is compensated by an increasing charge current conductance in $G_{\mathrm{EC}}$ $-G_{\mathrm{CA}}$, and there is no dip in the nonlocal differential conductance. At high energy, $G_{\mathrm{CA}}$ and $G_{\mathrm{DA}}$ vanish since the Andreev reflection rate vanishes.

\section{CONCLUSION}

In conclusion, we have studied nonlocal transport in a three-terminal device with two normal-metal terminals and one superconducting terminal. To this end we have applied the circuit theory of mesoscopic transport. The connectors between the circuit elements are represented by general expressions, relevant for a wide range of contacts. Dephasing is taken into account, and is governed by the inverse dwell time. This gives rise to an effective Thouless energy. For this model we have calculated the conductance associated with crossed Andreev reflection and electron cotunneling, as well as direct Andreev reflection. We find that for any contacts, electron cotunneling is the dominant nonlocal transport process. Results for various combinations of contacts of tunnel and metallic type are shown, and this demonstrates the strong dependence on the nature of the contacts. The conductance of nonlocal transport has a strong dependence on the Thouless energy. This is because, at these energies, dephasing leads to a loss of induced superconducting correlations. Several of the characteristics of our model have been observed in experiments, although we do not make complete qualitative contact to experimental data since electron cotunneling always dominates crossed Andreev reflection. We sug- gest determining the conductance due to these processes independently by carrying out additional energy transport measurements.

\section{ACKNOWLEDGMENTS}

This work was supported in part by The Research Council of Norway through Grants No. 167498/V30, No. 162742/ V00, No. 1534581/432, No. 1585181/143, and No. 1585471/ 431, RTN Spintronics, the Swiss NSF, the NCCR Nanoscience, the Deutsche Forschungsgemeinschaft through SFB 513, the Landesstiftung Baden-Württemberg through the Research Network "Functional Nanostructures," the National Science Foundation under Grant No. PHY99-07949, and the EU via Project No. NMP2-CT-2003-505587 "SFINx."

\section{APPENDIX: NOTATION}

Matrices in Nambu and Keldysh matrix space are denoted by a caret $(\hat{M})$ and an overbar $(\bar{M})$, respectively. The symbol

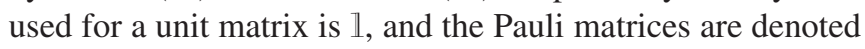
$\hat{\sigma}_{n}$ and $\bar{\tau}_{n}$ in Nambu and Keldysh space where $n=1,2,3$. Compositions of matrices in Nambu and Keldysh space are formed by a direct product to make up $4 \times 4$ matrices in Nambu-Keldysh matrix space, so that, e.g.,

$$
\hat{\sigma}_{3} \bar{\tau}_{1}=\left(\begin{array}{cccc}
0 & 0 & 1 & 0 \\
0 & 0 & 0 & -1 \\
1 & 0 & 0 & 0 \\
0 & -1 & 0 & 0
\end{array}\right) .
$$

*Electronic address: jan.morten@phys.ntnu.no

${ }^{1}$ J. M. Byers and M. E. Flatte, Phys. Rev. Lett. 74, 306 (1995).

${ }^{2}$ G. Deutsher and D. Feinberg, Appl. Phys. Lett. 76, 487 (2000).

${ }^{3}$ D. Beckmann, H. B. Weber, and H. v. Lohneysen, Phys. Rev. Lett. 93, 197003 (2004).

${ }^{4}$ S. Russo, M. Kroug, T. M. Klapwijk, and A. F. Morpurgo, Phys. Rev. Lett. 95, 027002 (2005).

${ }^{5}$ G. Falci, D. Feinberg, and F. W. J. Hekking, Europhys. Lett. 54, 255 (2001).

${ }^{6}$ T. Yamashita, S. Takahashi, and S. Maekawa, Phys. Rev. B 68, 174504 (2003).

${ }^{7}$ D. Sanchez, R. Lopez, P. Samuelsson, and M. Buttiker, Phys. Rev. B 68, 214501 (2003).

${ }^{8}$ N. M. Chtchelkatchev, JETP Lett. 78, 230 (2003).

${ }^{9}$ F. Giazotto, F. Taddei, R. Fazio, and F. Beltram, Phys. Rev. Lett. 97, 087001 (2006).

${ }^{10}$ P. Recher, E. V. Sukhorukov, and D. Loss, Phys. Rev. B 63, 165314 (2001).

${ }^{11}$ N. M. Chtchelkatchev, G. Blatter, G. B. Lesovik, and T. Martin, Phys. Rev. B 66, 161320(R) (2002).

${ }^{12}$ P. Recher and D. Loss, Phys. Rev. Lett. 91, 267003 (2003).

${ }^{13}$ F. W. J. Hekking and Y. V. Nazarov, Phys. Rev. B 49, 6847 (1994).

${ }^{14}$ D. Feinberg, Eur. Phys. J. B 36, 419 (2003).

${ }^{15}$ R. Mélin and D. Feinberg, Phys. Rev. B 70, 174509 (2004).
${ }^{16}$ R. Mélin, Phys. Rev. B 73, 174512 (2006).

${ }^{17}$ G. Bignon, M. Houzet, F. Pistolesi, and F. W. J. Hekking, Europhys. Lett. 67, 110 (2004).

${ }^{18}$ Y. V. Nazarov, Phys. Rev. Lett. 73, 1420 (1994).

${ }^{19}$ Y. V. Nazarov, Superlattices Microstruct. 25, 1221 (1999).

${ }^{20}$ A. Schmid and G. Schön, J. Low Temp. Phys. 20, 207 (1975).

${ }^{21}$ E. V. Bezuglyi, E. N. Bratus', V. S. Shumeiko, G. Wendin, and H. Takayanagi, Phys. Rev. B 62, 14439 (2000).

${ }^{22}$ W. Belzig, A. Brataas, Y. V. Nazarov, and G. E. W. Bauer, Phys. Rev. B 62, 9726 (2000).

${ }^{23}$ D. Huertas-Hernando, Y. V. Nazarov, and W. Belzig, Phys. Rev. Lett. 88, 047003 (2002).

${ }^{24}$ Y. Tanaka, Y. V. Nazarov, and S. Kashiwaya, Phys. Rev. Lett. 90, 167003 (2003)

${ }^{25}$ W. Belzig and Y. V. Nazarov, Phys. Rev. Lett. 87, 197006 (2001).

${ }^{26}$ W. Belzig and Y. V. Nazarov, Phys. Rev. Lett. 87, 067006 (2001).

${ }^{27}$ M. Vanevic and W. Belzig, Phys. Rev. B 72, 134522 (2005).

${ }^{28}$ J. Börlin, W. Belzig, and C. Bruder, Phys. Rev. Lett. 88, 197001 (2002).

${ }^{29}$ A. Brataas, G. E. W. Bauer, and P. J. Kelly, Phys. Rep. 427, 157 (2006).

${ }^{30}$ J. Rammer and H. Smith, Rev. Mod. Phys. 58, 323 (1986).

${ }^{31}$ T. H. Stoof and Y. V. Nazarov, Phys. Rev. B 53, 14496 (1996).

${ }^{32}$ W. Belzig, F. K. Wilhelm, C. Bruder, G. Schön, and A. D. Zaikin, Superlattices Microstruct. 25, 1251 (1999). 
${ }^{33}$ A. Schmid, in Nonequilibrium Superconductivity, Phonons, and Kapitza Boundaries, edited by Kenneth E. Gray, NATO Advanced Studies Institute, Series B: Physics (Plenum, New York, 1981), Vol. 65, pp. 423-480.

${ }^{34}$ D. Estève, H. Pothier, S. Guéron, and N. O. Birge, in Mesoscopic Electron Transport, edited by Lydia L. Sohn, Leo P. Kouwenhoven, and Gerd Schön, NATO Advanced Study Institute, Series E: Applied Science (Kluwer Academic, Dordrecht, 1997), Vol. 345, pp. 375-406.

${ }^{35}$ J. P. Morten, A. Brataas, and W. Belzig, Phys. Rev. B 70, 212508 (2004).

${ }^{36}$ J. P. Morten, A. Brataas, and W. Belzig, Phys. Rev. B 72, 014510 (2005).

${ }^{37}$ C. W. J. Beenakker, Phys. Rev. B 46, 12841 (1992).
${ }^{38}$ G. E. Blonder, M. Tinkham, and T. M. Klapwijk, Phys. Rev. B 25, 4515 (1982).

${ }^{39}$ C.-J. Chien and V. Chandrasekhar, Phys. Rev. B 60, 15356 (1999).

${ }^{40}$ The distribution functions $h_{\mathrm{T}}(E), h_{\mathrm{L}}(E)$ can be written in terms of the particle distribution function $f(E)$ as $h_{\mathrm{T}}=1-f(E)-f(-E)$ and $h_{\mathrm{L}}=-f(E)+f(-E)$ (see Ref. 32).

${ }^{41}$ To make contact with our notation we must identify $\alpha=\cos \theta$ and $\beta=\sin \theta$ in Eq. (II.29b) of Ref. 33. In addition to this, we put $\gamma=0$ since there is no such component in the retarded Green's function of the cavity, $\dot{\Phi}=0$ since we are considering stationary phenomena, and $\mathbf{v}_{\mathrm{S}}=\mathbf{0}$ since there is no supercurrent in the cavity. 